\title{
Resistance to Cucumber mosaic virus in Cowpea and Implications for Control of Cowpea Stunt Disease
}

\author{
A. G. Gillaspie, Jr., USDA-ARS, Plant Genetic Resources Conservation Unit, Griffin, GA 30223
}

\begin{abstract}
Gillaspie, A. G., Jr. 2001. Resistance to Cucumber mosaic virus in cowpea and implications for control of cowpea stunt disease. Plant Dis. 85:1004-1005.

Cucumber mosaic virus (CMV) and Blackeye cowpea mosaic virus (BICMV) interact synergistically in dually infected plants of cowpea (Vigna unguiculata subsp. unguiculata) to cause cowpea stunt disease, the most damaging viral disease of this crop in the U.S. Sources of resistance to BlCMV are known and are present in cultivars of cowpea such as Pinkeye Purple HullBVR. However, no sources of CMV resistance have been found previously in cowpea. In 1998, PI 441918, a cowpea line growing in regeneration plots, was observed to have few viral symptoms, was not infected with BlCMV, and had a low titer of CMV when tested using direct antigen coating-enzyme-linked immunosorbent assay (DAC-ELISA). In greenhouse tests, infection of PI 441918 with CMV resulted in a lower titer of virus if the inoculated plants were from white seeds of PI 441918 than if the plants were from tan seeds of this PI, and a lower titer of virus than plants of the susceptible cultivar Coronet. In the field, with CMV- and BICMVinfected plants of Coronet in spreader rows, plants from white seed of PI 441918 had no infection with BlCMV and a low infection rate with CMV. PI 441918 offers a high level of resistance to BlCMV and moderate resistance to CMV, which are important characteristics in a parental line to develop cultivars of cowpea resistant to cowpea stunt disease.
\end{abstract}

Cucumber mosaic virus (CMV) causes some degree of distortion and a green mosaic on the leaves of many lines of cowpea (Vigna unguiculata (L.) Walp. subsp. unguiculata). This virus has a very large host range, causes a variety of symptoms on different genotypes of cowpeas, and has potential for seed transmission as well as aphid transmission (2,3). Blackeye cowpea mosaic virus (BlCMV) causes mild to moderate mosaic symptoms on leaves of cowpea. These two viruses interact synergistically in cowpea to cause cowpea stunt disease $(1,8)$. Cowpea stunt causes significant losses in production of cowpea in the United States. Leaves of the infected plants are small, mottled, blistered, and distorted. Severity of the symptoms varies depending on the line of cowpea and the strain of CMV.

The Plant Genetic Resources Conservation Unit at Griffin, GA, part of the National Plant Germplasm System, is responsible for the acquisition, maintenance, evaluation, and distribution of germplasm such as Vigna to breeders and other researchers in the United States and elsewhere. A portion of the maintenance in-

Corresponding author: A. G. Gillaspie, Jr.

E-mail: s9gg@ars-grin.gov

Accepted for publication 3 June 2001 .

Publication no. D-2001-0703-01R

This article is in the public domain and not copyrightable. It may be freely reprinted with customary crediting of the source. The American Phytopathological Society, 2001. volves replenishment of seed stocks when the quantities or the viabilities become limiting. Cowpea is a self-pollinating crop, with a very low degree of outcrossing present with some lines and a crop that grows in tropical and subtropical regions requiring soil temperatures above $50^{\circ} \mathrm{C}$. Several strains of CMV are present in Georgia (4), where much of the regeneration of cowpea germ plasm occurs and cowpea stunt is a major problem. Cowpea stunt appears to limit seed production in some lines of cowpea, and both viruses are seedtransmitted in cowpea. Therefore, the two viruses pose a problem for seed production and distribution of germ plasm. Sources of resistance to BlCMV are known and are present in cowpea cultivars such as Pinkeye Purple Hull-BVR. Varying degrees of resistance or tolerance to CMV have been suggested to exist in several cowpea cultivars, but no reliable source of resistance to CMV isolates involved in cowpea stunt has been identified $(2,6,7)$. McWilliams screened 350 lines of cowpeas from the National Plant Germplasm Collection in greenhouse tests and found four lines that may have promise, but these have not been tested under field conditions with aphid transmission (7).

The USDA cowpea regeneration plots in Griffin, GA, provide a natural screen for cowpea stunt. In 1998, the cowpea line PI 441918 from Brazil stood out among all the cowpea lines in the regeneration plots as green, healthy-looking plants late in the season, in contrast to lines in other plots that were diseased and stunted to various degrees. This paper describes the discovery of a line of cowpea that displays resistance to CMV and BlCMV.

\section{MATERIALS AND METHODS}

All the plants of PI 441918 in the regeneration plot were tested for the presence of CMV and BlCMV by direct antigen coating-enzyme-linked immunosorbent assay (DAC-ELISA) using polyclonal rabbit antisera. The BICMV antiserum (supplied by J. W. Demski, University of Georgia, Griffin) was diluted to 1:20,000. The CMV antiserum (supplied by O. W. Barnett, North Carolina State University, Raleigh) was diluted to 1:5,000. A leaflet from the youngest trifoliolate was collected from each plant and disks were removed from each leaflet with a no. 13 core borer. The disks were ground in extraction buffer and processed as described previously (5). Absorbance was measured using an Emax Microplate Reader (Molecular Dynamics, Sunnyvale, CA). Plants of PI 441918 had little (1/19) CMV and no BlCMV when tested by ELISA.

PI 441918 is a mixed line that produces two distinct types of seed, almost white seed and tan seed. For a preliminary test, seeds of PI 441918 were separated by color, 100 seed of each color and of a susceptible control (Coronet) were planted in flats (50 seed per flat) in a greenhouse, and mechanically inoculated with CMV obtained from infected cowpea, which had been inoculated with a Georgia strain of the virus (4). Disease occurrence was judged by the presence of symptoms and by DAC-ELISA of each plant at the thirdtrifoliolate stage.

A screening test (Test I) was done in the field the Griffin from June 1 (planting) to August 6 (harvest), 1999, with CMV-inoculated spreader rows of Coronet. A randomized complete block design was used with four replications of whiteseeded PI 441918, tan-seeded PI 441918, and Coronet. Each single row plot was 3 $\mathrm{m}$ long with a $3 \mathrm{~m}$ row spacing and $3 \mathrm{~m}$ alleys. All treatment rows were adjacent to a spreader row. The plants for the spreader rows were mechanically inoculated in the greenhouse and tested by ELISA for infection with CMV. Three weeks after inoculation with CMV, the infected plants were hand-transplanted (12 to 15 plants per single-row plot) in the field. Healthy plants (12 to 15 per replication) of the test lines were grown in the greenhouse for 4.5 weeks and transplanted on the same day as the spreader row plants. 
Table 1. DAC-ELISA ratings in field tests of cowpea for susceptibility to Cucumber mosaic virus

\begin{tabular}{lcc}
\hline Cowpea line & Positive plants/total plants & Mean $^{\mathbf{a}}$ \\
\hline Test I, 1 June-6 August 1999 & & \\
PI 441918-white seed & $23 / 45$ & 0.516 \\
PI 441918-tan seed & $32 / 45$ & 0.711 \\
Coronet & $44 / 47$ & 0.935 \\
LSD 0.01 & & 0.213 \\
Test II, 5 June-25 July 2000 & & \\
PI 441918-white seed & $10 / 30$ & 0.304 \\
Coronet & $22 / 22$ & 1.000 \\
LSD 0.01 & & 0.551 \\
Test III, 17 August-19 September 2000 & & \\
PI 441918-white seed & $6 / 43$ & 0.133 \\
Coronet & $69 / 78$ & 0.870 \\
LSD & & 0.167 \\
\hline
\end{tabular}

${ }^{\text {a }}$ Mean across four blocks of positive plants/randomized block.

Test II was run from June 5 to July 25 , 2000, and Test III was run from August 17 to September 19, 2000, in a field in Griffin. The spreader rows of Coronet plants alternated plants infected with CMV with plants infected with BlCMV. These spreader row plants were mechanically inoculated in the greenhouse, tested for CMV and BICMV by DAC-ELISA, and transplanted into the field 3 weeks after being inoculated. These screening tests were arranged as were those in Test I, using PI 441918-white seed and Coronet as treatments, but these test plots were directseeded on the same day as the spreader row plants were transplanted.

Infection data for each virus were collected at the end of all three tests using DAC-ELISA of each plant in the test. The data were subjected to analysis of variance and mean comparisons with least significant differences, using PC-SAS for Windows Version 6.12 (SAS, Cary, NC).

\section{RESULTS AND DISCUSSION}

In the preliminary greenhouse test, $78 \%$ (77/91) of the CMV-inoculated plants of PI 441918-tan seed were infected, 76\% (66/87) of the PI 441918-white seed plants were infected, and 95\% (76/80) of the susceptible cv. Coronet plants were infected, based on results of DAC-ELISA. Mean $\mathrm{A}_{405}$ readings were 1.0, 0.4, and 1.3, respectively, suggesting that plants of PI 441918-white seed may have supported less viral multiplication than plants of PI 441918-tan seed or of Coronet.

Results of the Test I (Table 1) indicated that the plants of PI 441918-white seed had significantly less infection with CMV than Coronet, but were not significantly different from plants of PI 441918-tan seed. The plants of PI 441918-tan seed were significantly less infection than Coronet, but the difference was not as great as found for the plants grown from white seeds. The BlCMV screen test in 1999 showed that plants of PI 441918-white seed were not infected.

In 2000, Test II and Test III, in which the spreader rows of Coronet consisted of plants inoculated with CMV and plants with inoculated BlCMV, produced results similar to Test I (Table 1). In Test II, none of the plants (out of 30) of PI 441918-white seed and 19 plants (out of 22) of Coronet were infected with BlCMV. Similarly, not one of the 43 plants of PI 441918-white seed and 62 plants (out of 78) of Coronet were infected with BlCMV in Test III. Plants of PI 441918-white seed differed in susceptibility to both CMV and BlCMV compared to plants of the cowpea cultivar Coronet, in all three field tests.

Plants of PI 441918-white and tan seed were highly resistant to BlCMV and had significantly less CMV infection than Coronet. Although only single strains of BlCMV and CMV were inoculated onto the spreader row plants, there were several other strains endemic in the area of these field trials (unpublished data). Therefore, the resistance present in PI 441918 may be broad enough to reduce infection by the Georgia strains of the viruses. It is not known how well the resistance will hold up with mixed virus strains in other production areas such as Arkansas where cowpea stunt disease is also a problem (1).

The lower incidence of infection in the 2000 field trials compared to the 1999 field test probably was associated the shorter times between plantings and the final readings of the 2000 field tests. Virus titer and incidence increase with the duration of infection. Plants of PI 441918-white seed only produced white seeds each time it was planted (unpublished data). Plants of PI 441918-white seed offer the very high level of resistance to BlCMV and moderate resistance to CMV under conditions of this study. These are valuable traits for development of cultivars of cowpea more resistant to cowpea stunt disease. This line will be usable by U.S. breeders because it flowers in Georgia, has a 105-day maturity, and an average seed weight. To our knowledge, this is the first report of resistance in cowpea to CMV.

Further study is needed to determine how useful this resistance is when challenged with strains or isolates from other areas. This would be best done by sending seeds to be tested in other growing areas rather than risking introduction of new strains into Georgia. Also, information on seed transmission rates of the resistant line should be obtained because we found only one case of seed transmission, but no testing has been performed.

\section{ACKNOWLEDGMENTS}

The author thanks James Chalkley for bringing attention to PI 441918 in the regeneration plot and for technical assistance. The author also thanks D. L. Pinnow for technical assistance with the ELISA testing, and R. Dean and J. Davis for help with statistical analysis.

\section{LITERATURE CITED}

1. Anderson, E. J., Kline, A. S., Kim, K. S. Goeke, S. C., and Albritton, C. W. 1994. Identification of cowpea stunt disease in south central Arkansas. Ark. Farm Res. 43:14-15.

2. Anderson, E. J., Kline, A. S., Morelock, T. E., and McNew, R. W. 1996. Tolerance to Blackeye cowpea mosaic potyvirus not correlated with decreased virus accumulation or protection from cowpea stunt disease. Plant Dis. 80:847-852

3. Francki, R. I. B., Mossop, D. W., and Hatta, T. 1979. Cucumber mosaic virus. CMI/AAB Descriptions of Plant Viruses. No. 213.

4. Gillaspie, A. G., Jr., Hajimorad, M. R., and Ghabrial, S. A. 1998. Characterization of a severe strain of cucumber mosaic cucumovirus seedborne in cowpea. Plant Dis. 82:419-422.

5. Gillaspie, A. G., Jr., Hopkins, M. S., Pinnow, D. L., and Hampton, R. O. 1995. Seedborne viruses in preintroduction cowpea seed lots and establishment of virus-free accessions. Plant Dis. 79:388-391.

6. Kuhn, C. W., Brantley, B. B., and Sowell, G., Jr. 1966. Southern pea viruses: identification, symptomatology, and sources of resistance. Georgia Agric. Exp. Stn. Bull. N. S. 157.

7. McWilliams, J. D. 1998. Screening a cowpea germplasm collection for resistance to cowpea stunt-causing viruses. M. S. thesis, University of Arkansas, Fayetteville.

8. Pio-Ribeiro, G., Wyatt, S. D., and Kuhn, C. W. 1978. Cowpea stunt: a disease caused by a synergistic interaction of two viruses. Phytopathology 68:1260-1265. 